\title{
FORMALLY REAL INVOLUTIONS ON CENTRAL SIMPLE ALGEBRAS
}

\author{
J. CIMPRIČ
}

\begin{abstract}
An involution \# on an associative ring $R$ is formally real if a sum of nonzero elements of the form $r^{\#} r$ where $r \in R$ is nonzero. Suppose that $R$ is a central simple algebra (i.e. $R=$ $M_{n}(D)$ for some integer $n$ and central division algebra $\left.D\right)$ and \# is an involution on $R$ of the form $r^{\#}=a^{-1} r^{*} a$, where $*$ is some transpose involution on $R$ and $a$ is an invertible matrix such that $a^{*}= \pm a$. In section 1 we characterize formal reality of \# in terms of $a$ and $\left.*\right|_{D}$. In later sections we apply this result to the study of formal reality of involutions on crossed product division algebras. We can characterize involutions on $D=(K / F, \Phi)$ that extend to a formally real involution on the split algebra $D \otimes_{F} K \cong M_{n}(K)$. Every such involution is formally real but we show that there exist formally real involutions on $D$ which are not of this form. In particular, there exists a formally real involution \# for which the hermitian trace form $x \mapsto \operatorname{tr}\left(x^{\#} x\right)$ is not positive semidefinite.
\end{abstract}

\section{1. $\epsilon$-HERMITIAN CONES ON CENTRAL SIMPLE ALGEBRAS}

We say that an involution $*$ on a central simple algebra $R$ is formally real if any finite sum of nonzero elements of the form $r r^{*}$ where $r \in R$ is nonzero. In this section we introduce our main technical tool for the study of formally real involutions - the notion of an $\epsilon$-hermitian cone. The precise relationship between $\epsilon$-hermitian cones and formally real involutions is explained by Corollary 4 .

Recall that a central simple algebra is a full matrix ring over a central division algebra. Let $R$ be a central simple $F$-algebra with involution * and $\epsilon \in F$ such that $\epsilon \epsilon^{*}=1$. An element $a \in R$ is $\epsilon$-hermitian if $\epsilon a^{*}=a$. The set of all $\epsilon$-hermitian elements in $R$ will be denoted by $S_{\epsilon}(R)$. A subset $M$ of $S_{\epsilon}(R)$ such that $M+M \subseteq M, a M a^{*} \subseteq M$ for every $a \in R$ and $M \cap-M=\{0\}$ will be called an $\epsilon$-hermitian cone on $R$.

Date: August 10th 2006, revised January 28th 2007.

2000 Mathematics Subject Classification. 16K20, 16W10, $12 \mathrm{D} 15$.

Key words and phrases. involutions, central simple algebras, crossed products, sums of hermitian squares. 
Theorem 1. Let $D$ be a central division F-algebra with involution *. For every $\epsilon \in F$ such that $\epsilon \epsilon^{*}=1$ and for every integer $n$ there exists a one-to-one correspondence between

- $\epsilon$-hermitian cones on $D$ and

- $\epsilon$-hermitian cones on $M_{n}(D)$ (with involution $\left[x_{i j}\right]^{*}=\left[x_{j i}^{*}\right]$ ).

We will need the following well-known "Diagonalization Theorem".

Proposition 2. Every matrix $A \in S_{\epsilon}(R)$ is congruent to a direct sum of matrices of the form $[a]$ or $\left[\begin{array}{cc}0 & b \\ \epsilon b^{*} & 0\end{array}\right]$ where $a \in S_{\epsilon}(D)$ and $b \in D$. Moreover, if either $\epsilon \neq-1$ or $\left.*\right|_{D} \neq \mathrm{id}$ then every matrix of the form $\left[\begin{array}{cc}0 & b \\ \epsilon b^{*} & 0\end{array}\right]$ is congruent to a diagonal matrix.

Proof. The first claim is proved by induction on $n$ using the identity

$$
\left[\begin{array}{cc}
I & 0 \\
-B_{21} B_{11}^{-1} & I
\end{array}\right]\left[\begin{array}{cc}
B_{11} & B_{12} \\
B_{21} & B_{22}
\end{array}\right]\left[\begin{array}{cc}
I & -B_{11}^{-1} B_{12} \\
0 & I
\end{array}\right]=\left[\begin{array}{cc}
B_{11} & 0 \\
0 & \tilde{B}_{11}
\end{array}\right]
$$

where $\tilde{B}_{11}=B_{22}-B_{21} B_{11}^{-1} B_{12}$ is the Schur complement of $B_{11}$. The second claim is just a short computation.

Now, we can prove the theorem.

Proof. Let us start with the case $\epsilon=-1$ and $\left.*\right|_{D}=\mathrm{id}$. In this case $D=F$ and $2 S_{\epsilon}(D)=0$. Let $M$ be an $\epsilon$-hermitian cone on $M_{n}(D)$. Pick any $C \in M$. By Proposition 2, there exists an invertible matrix $P$ such that

$$
P^{*} C P=\bigoplus_{i=1}^{r}\left[a_{i}\right] \oplus \bigoplus_{j=1}^{s}\left[\begin{array}{cc}
0 & b_{j} \\
-b_{j} & 0
\end{array}\right],
$$

where $a_{i} \in S_{\epsilon}(D)$ and $b_{j} \in D$. Note that the matrix

$$
Q=\bigoplus_{i=1}^{r}[1] \oplus \bigoplus_{j=1}^{s}\left[\begin{array}{ll}
0 & 1 \\
1 & 0
\end{array}\right]
$$

is invertible and $Q^{*} P^{*} C P Q=-P^{*} C P$. The latter follows from

$$
\left[a_{i}\right]=-\left[a_{i}\right] \text { and } \quad\left[\begin{array}{ll}
0 & 1 \\
1 & 0
\end{array}\right]\left[\begin{array}{cc}
0 & b_{j} \\
-b_{j} & 0
\end{array}\right]\left[\begin{array}{ll}
0 & 1 \\
1 & 0
\end{array}\right]=-\left[\begin{array}{cc}
0 & b_{j} \\
-b_{j} & 0
\end{array}\right] \text {. }
$$

It follows that $P^{*} C P \in M \cap-M=\{0\}$, so that $C=0$. Hence $\{0\}$ is the only $\epsilon$-hermitian cone on $M_{n}(D)$. For $n=1$ we get that $\{0\}$ is also the only $\epsilon$-hermitian cone on $D$.

From now on we assume that either $\epsilon \neq-1$ or $\left.*\right|_{D} \neq$ id. Therefore, every $\epsilon$-hermitian matrix is congruent to a diagonal matrix. 
For every $\epsilon$-hermitian cone $N$ of $D$ write

$$
F(N)=\left\{A \in S_{\epsilon}\left(M_{n}(D)\right) \mid x A x^{*} \in N \text { for every } x \in D^{n}\right\} .
$$

We claim that $F(N)$ is an $\epsilon$-hermitian cone on $M_{n}(D)$. If $A, B \in F(N)$, then $x(A+B) x^{*}=x A x^{*}+x B x^{*} \in N$ for every $x \in D^{n}$. Hence $A+B \in F(N)$. If $A \in F(N)$ and $B \in M_{n}(D)$, then $x B A B^{*} x^{*}=$ $(x B) A(x B)^{*} \in N$ for every $x \in D^{n}$. Hence $B A B^{*} \in F(N)$. If $N$ is proper, then $F(N)$ is also proper. Namely, if $A \in F(N) \cap-F(N)$, then $x A x^{*} \in N \cap-N=\{0\}$ for every $x \in D^{n}$. Let $A^{\prime}$ be the diagonal matrix congruent to $A$. Then $x A^{\prime} x^{*}=0$ for every $x \in D^{n}$, so that $A^{\prime}=0$. Hence, $A=0$.

For every $\epsilon$-hermitian cone $M$ on $M_{n}(D)$ write

$$
G(M)=\left\{c \in S_{\epsilon}(D) \mid c E_{11} \in M\right\} .
$$

We claim that $G(M)$ is an $\epsilon$-hermitian cone on $D$. If $a, b \in G(M)$, then $(a+b) E_{11}=a E_{11}+b E_{11} \in M$, hence $a+b \in G(M)$. If $a \in$ $G(M)$ and $d \in D$ then $\left(d a d^{*}\right) E_{11}=\left(d E_{11}\right)\left(a E_{11}\right)\left(d E_{11}\right)^{*} \in M$, hence $d a d^{*} \in G(M)$. If $M$ is proper, then $G(M)$ is also proper. Namely, if $a \in G(M) \cap-G(M)$, then $a E_{11} \in M \cap-M=\{0\}$. Hence $a E_{11}=0$.

We claim that for every $\epsilon$-hermitian cone $N$ on $D$ we have that $G(F(N))=N$. If $a \in G(F(N))$, then $a E_{11} \in F(N)$. Then $x\left(a E_{11}\right) x^{*} \in$ $N$ for every $x \in D^{n}$. In particular, for $x=(1,0, \ldots, 0)$, we get that $a \in N$. To prove the opposite inclusion pick any $a \in N$. Then $a E_{11} \in$ $F(N)$ since $x\left(a E_{11}\right) x^{*}=x_{1} a x_{1}^{*} \in N$ for every $x \in D^{n}$. It follows that $a \in G(F(N))$.

We claim that for every $\epsilon$-hermitian cone $M$ on $M_{n}(D)$ we have that $F(G(M))=M$. Since every $\epsilon$-hermitian matrix is congruent to a diagonal matrix, it suffices to show that a diagonal matrix belongs to $F(G(M))$ if and only if it belongs to $M$. Pick a diagonal matrix $A \in F(G(M))$. Then for every $x \in D^{n}, x A x^{*} \in G(M)$. In particular $a_{11}, \ldots, a_{n n} \in G(M)$. It follows that $a_{11} E_{11}, \ldots, a_{n n} E_{11} \in M$. Hence $A=\sum_{j} P_{1 j}\left(a_{j j} E_{11}\right) P_{1 j}^{*} \in M$. To prove the opposite inclusion, pick $A \in M$. It follows that $a_{j j} E_{11}=E_{1 j} A E_{1 j}^{*} \in M$ for every $j$. Hence $a_{11}, \ldots, a_{n n} \in G(M)$. It follows that $x A x^{*}=\sum_{j} x_{j} a_{j j} x_{j}^{*} \in G(M)$ for every $x \in D^{n}$. Hence $A \in F(G(N))$.

Let $R=M_{n}(D)$ be a central simple $F$-algebra with char $F \neq 2$. The following is a summary of [4, Theorem 3.1 and Proposition 2.20: For every involution \# on $M_{n}(D)$ there exists an involution $*$ on $D$ such that $\left.\#\right|_{F}=\left.*\right|_{F}$ and an invertible matrix $A \in M_{n}(D)$ such that $A^{*}= \pm A$ and $X^{\#}=A^{-1} X^{*} A$ for every matrix $X \in M_{n}(D)$ (where $\left.\left[x_{i j}\right]^{*}=\left[x_{j i}^{*}\right]\right)$. If $\left.*\right|_{F} \neq$ id we can assume $A^{*}=A$.

Our next result extends Theorem 1 to arbitrary involutions. 
Theorem 3. Let $D$ be a central division $F$-algebra with involution * and $\eta \in F$ such that $\eta \eta^{*}=1$. Let $n$ be an integer and $A \in S_{\eta}\left(M_{n}(D)\right)$ an invertible matrix. Then $X^{\#}=A^{-1} X^{*} A$ is also an involution on $M_{n}(D)$. For every $\epsilon \in F$ such that $\epsilon \epsilon^{*}=1$ there exists a one-to-one correspondence between:

(1) $\epsilon$-hermitian cones on $\left(M_{n}(D), \#\right)$,

(2) $\epsilon \eta$-hermitian cones on $\left(M_{n}(D), *\right)$,

(3) $\epsilon \eta$-hermitian cones on $D$.

Proof. Let $\phi: M_{n}(D) \rightarrow M_{n}(D)$ be the mapping defined by $\phi(X)=$ $A X$. A short computation shows that $\phi$ induces a one-to-one correspondence between the set $S_{\epsilon}\left(M_{n}(D)\right.$, \#) and the set $S_{\epsilon \eta}\left(M_{n}(D)\right.$, *). Moreover $\phi$ is additive and for every $X \in S_{\epsilon}\left(M_{n}(D)\right.$, \#) and $Y \in M_{n}(D)$ we have that $\phi\left(Y^{\#} X Y\right)=Y^{*} \phi(X) Y$. Therefore the mappings $M \rightarrow \phi(M)$ and $N \rightarrow \phi^{-1}(N)$ give a one-to-one correspondence between (1) and (2) for every $\epsilon$. The mappings $F$ and $G$ from Theorem 1 give a one-to-one correspondence between (2) and (3).

As a corollary of Theorem 3 , we obtain a characterization of formally real involutions on central simple algebras.

Corollary 4. Let $D, F, *, \eta, n, A, \#$ be as in Theorem 3. If either $\eta \neq$ -1 or $\left.*\right|_{D} \neq \mathrm{id}$, then the following assertions are equivalent:

(1) \# is formally real,

(2) there exists a 1-hermitian cone on $\left(M_{n}(D)\right.$,\#) which contains the identity matrix I,

(3) there exists an $\eta$-hermitian cone on $\left(M_{n}(D), *\right)$ which contains the matrix A,

(4) there exists an $\eta$-hermitian cone on $(D, *)$ which contains all elements in some diagonal representation of $A$.

If $\eta=-1$ and $\left.*\right|_{D}=$ id then $\#$ is not formally real.

A 1-hermitian cone containing 1 will be called unital hermitian cone in the sequel. If $A$ has a nonzero hermitian square on its diagonal (e.g. 1) then every 1-hermitian cone on $\left(M_{n}(D), *\right)$ which contains the matrix $A$ is a unital hermitian cone. If $D$ is a division algebra admitting a unital hermitian cone then char $K=0$ for every subfield $K$ of $D$.

\section{Extensions of involutions from $D$ to $D \otimes K$.}

Let $D$ be a central division $F$-algebra and $K$ a maximal subfield of $D$. Every involution $*$ on $D$ such that $K^{*} \subseteq K$ extends to an involution on $D \otimes_{F} K$. This is clear if $*$ is of the first kind (i.e. $\left.*\right|_{F}=$ id). If $*$ is of the second kind (i.e. $\left.*\right|_{F} \neq \mathrm{id}$ ), one has to observe first that 
$D \otimes_{F} K$ is isomorphic to $D \otimes_{F_{0}} K_{0}$ where $K_{0}=S_{1}(K)=\operatorname{Sym}(K)$ and $F_{0}=S_{1}(F)=\operatorname{Sym}(F)$ are the symmetric parts of $K$ and $F$. The aim of this section is to give an explicit construction of the extension which will be used in later sections. From now on we assume that char $K=0$.

Lemma 5. For every involution * on D there exists a maximal (i.e. self-centralizing) subfield of $D$ which is $*$-invariant.

Proof. We need the following claim: A central simple algebra with involution in which every normal element is central is a field.

Let $A$ be a central simple algebra in which every normal element is central. Pick any element $a \in A$. The element $\alpha=a+a^{*}$ is symmetric, hence normal. By the assumption $\alpha \in Z(A)$. It follows that $a a^{*}=a(\alpha-a)=(\alpha-a) a=a^{*} a$. Since $a$ is normal, it is central by the assumption. Hence $A=Z(A)$.

Suppose now that $L$ is a $*$-subfield of a division algebra $D$ which is not contained in any other $*$-subfield. Its centralizer $A=C_{D}(L)$ is also *-invariant and it contains $L$. By the Double Centralizer Theorem, $A$ is a simple algebra with $Z(A)=L$. If $A \neq L$, then $A$ contains a noncentral normal element $d$ by the claim. Then $L\left(d, d^{*}\right)$ is also a $*$-subfield of $D$ properly containing $L$, contrary to the choice of $L$. Therefore $A=L$.

Lemma 6. Every finite extension of fields with involution is generated by either a symmetric or an antisymmetric element. (We assume that both fields have characteristic zero.)

Proof. Let $K / F$ be a finite extension of fields with involution. This means that $K$ is a field with involution $*, F^{*} \subseteq F$ and $K / F$ is a finite field extension. Write $F_{0}=\operatorname{Sym}(F)$ and $K_{0}=\operatorname{Sym}(K)$. By the Primitive Element Theorem, there exists an element $\theta$ such that $K=F_{0}(\theta)$. If $K=K_{0}$ then $\theta$ is symmetric and we are done. If $K \neq K_{0}$, then $K=F_{0}\left(\theta-\theta^{*},\left(\theta-\theta^{*}\right)\left(\theta+\theta^{*}\right)\right)$ and both generators are antisymmetric. By the Primitive Element Theorem, there exists an $F_{0}$-linear combination $\theta^{\prime}$ of them such that $K=F_{0}\left(\theta^{\prime}\right)$. Hence, $\theta^{\prime}$ is antisymmetric and $K=F\left(\theta^{\prime}\right)$. It is also interesting to note that if $F \neq F_{0}$ then $K / F$ is generated by a symmetric element. Namely, $K=F\left(k \theta^{\prime}\right)$, where $k$ is an antisymmetric generator of $F / F_{0}$.

Proposition 7. Let $K$ be a maximal *-subfield of a division algebra $D$. There exists a unital, hermitian and $K-K$ bilinear mapping $f: D \rightarrow$ $K$.

Proof. Let $D$ be a division algebra with center $F$ and $K$ a maximal *-subfield in $D$. Pick a symmetric or antisymmetric element $x \in K$ 
such that $K=F_{0}(x)$ and let $\chi(t)=t^{n}+a_{n-1} t^{n-1}+\ldots+a_{1} t+a_{0}$ be its minimal $F_{0}$-polynomial. Write

$$
\begin{aligned}
& y_{0}=a_{0} x^{n-1} \\
& y_{1}=a_{0} x^{n-2}+a_{1} x^{n-1}, \\
& y_{2}=a_{0} x^{n-3}+a_{1} x^{n-2}+a_{2} x^{n-1}, \\
& \vdots \\
& y_{n-2}=a_{0} x+a_{1} x^{2}+a_{2} x^{3}+\ldots+a_{n-2} x^{n-1}, \\
& y_{n-1}=a_{0}+a_{1} x+a_{2} x^{2}+\ldots+a_{n-2} x^{n-2}+a_{n-1} x^{n-1}=-x^{n} .
\end{aligned}
$$

The mapping $f$ is defined by

$$
f(z)=\frac{-1}{\chi^{\prime}(x) x^{n}} \sum_{i=0}^{n-1} x^{i} z y_{i} .
$$

The coefficient was chosen so that $f(1)=1$ as one can easily verify. Clearly, $f$ is $K-K$ bilinear. A short computation shows that for every $z \in D, x f(z)=f(z) x$. This relation implies that $f(z)$ commutes with all elements from $K$, hence it belongs to $K$. To prove that $f\left(z^{*}\right)=f(z)^{*}$ for every $z \in D$ one has to distinguish the case when $x$ is symmetric from the case when $x$ is antisymmetric. The symmetric case is easy. In the antisymmetric case we use the fact that $\chi(t)$ has only even powers.

Remark. Note that $\operatorname{tr}=\operatorname{tr}_{K / F} \circ f$, where $\operatorname{tr}$ is the reduced trace.

Let $K$ be a maximal subfield of a division algebra $D$ and $e_{1}, \ldots, e_{n}$ a right $K$-basis of $D$. Let $\lambda: D \rightarrow M_{n}(K)$ be the left regular representation defined by $a\left[e_{1}, \ldots, e_{n}\right]=\left[e_{1}, \ldots, e_{n}\right] \lambda(a)$ for $a \in D$ and let $j: K \rightarrow M_{n}(K)$ be the natural imbedding defined by $j(k)=k I$ where $I \in M_{n}(K)$ is the identity matrix. Then the mapping $D \otimes_{F} K \rightarrow$ $M_{n}(K)$ defined by $a \otimes k \mapsto \lambda(a) j(k)$ is an isomorphism.

Proposition 8. Setup from above. Let $*$ be an involution on $D$ which leaves $K$ invariant and let $\left[a_{i j}\right]^{*}=\left[a_{j i}^{*}\right]$ be its extension to $M_{n}(K)$. Then the matrix $A=\left[f\left(e_{i}^{*} e_{j}\right)\right]_{i, j=1, \ldots, n} \in M_{n}(K)$ is nonsingular and hermitian (i.e. $A^{*}=A$ ) and the involution \# on $M_{n}(K)$ defined by $X^{\#}=A^{-1} X^{*} A$ satisfies $\lambda\left(a^{*}\right)=\lambda(a)^{\#}$ for every $a \in D$.

Proof. Since $f$ is hermitian, it follows that $A$ is hermitian. If $A$ is singular, then there exists a vector $v=\left(\alpha_{1}, \ldots, \alpha_{n}\right) \in K^{n}$ such that $A v=0$. Since $f$ is right $K$-linear, it follows that $f\left(e_{i}^{*}\left(\sum_{j=1}^{n}\left(e_{j} \alpha_{j}\right)\right)=0\right.$ for every $i$. Since $f$ is left $K$-linear, it follows that $f\left(\left(\sum_{i=1}^{n} e_{i} \beta_{i}\right)^{*}\left(\sum_{j=1}^{n} e_{j} \alpha_{j}\right)\right)=$ 0 for any $\beta_{1}, \ldots, \beta_{n} \in K$. Since $D$ is a division algebra we can pick 
$\beta_{i}$ so that $\left(\sum_{i=1}^{n} e_{i} \beta_{i}\right)^{*}\left(\sum_{j=1}^{n} e_{j} \alpha_{j}\right)=1$. This is a contradiction with $f(1)=1$. Hence $A$ is nonsingular. By the definition of $\lambda$, we get that

$\lambda(a)^{*}\left[\begin{array}{cc}e_{1}^{*} e_{1} & e_{1}^{*} e_{n} \\ e_{n}^{*} e_{1} & e_{n}^{*} e_{n}\end{array}\right]=\left[\begin{array}{c}e_{1}^{*} \\ e_{n}^{*}\end{array}\right] a^{*}\left[\begin{array}{ll}e_{1} & e_{n}\end{array}\right]=\left[\begin{array}{cc}e_{1}^{*} e_{1} & e_{1}^{*} e_{n} \\ e_{n}^{*} e_{1} & e_{n}^{*} e_{n}\end{array}\right] \lambda\left(a^{*}\right)$

for every $a \in D$. Applying $f$ to all elements of this identity we get that $\lambda\left(a^{*}\right)=A^{-1} \lambda(a)^{*} A=\lambda(a)^{\#}$ for every $a \in D$.

Example 1. Let $D=\left(\frac{a, b}{F}\right)$ be a quaternion algebra. Recall that $D$ is an $F$-algebra with two generators $i$ and $j$, and three relations $i^{2}=a, j^{2}=b, i j=-j i$. It is a division algebra if and only if the only solution in $F$ of the equation $a x^{2}+b y^{2}=z^{2}$ is $x=y=z=0$. If $z=\alpha+\beta i+\gamma j+\delta k$ then $f(z)=\alpha+\beta i$. (Take $x=i, \chi(t)=t^{2}-a, y_{0}=$ $-a i, y_{1}=-a, n=2$ in the definition of $f$.) Let $*$ be an involution of $D$ defined by $i^{*}=i$ and $j^{*}=j$. Write $k=i j$. Then $k^{*}=-k$ and $1, i, j, k$ is an $F$-basis of $D$. The subfield $K=F(i)$ is maximal and $*$-invariant. Note that $1, j$ is a right $K$-basis of $D$ and

$$
A=\left[\begin{array}{ll}
f\left(1^{*} 1\right) & f\left(1^{*} j\right) \\
f\left(j^{*} 1\right) & f\left(j^{*} j\right)
\end{array}\right]=\left[\begin{array}{ll}
1 & 0 \\
0 & b
\end{array}\right]=\left[\begin{array}{cc}
a_{1} & 0 \\
0 & a_{2}
\end{array}\right]
$$

With respect to this basis, the imbedding $\lambda: D \rightarrow M_{2}(K)$ is given by

$$
\lambda(z)=\left[\begin{array}{cc}
\alpha+\beta i & b(\gamma+\delta i) \\
\gamma-\delta i & \alpha-\beta i
\end{array}\right]
$$

The involution \# on $M_{2}(K)$ which extends the involution $*$ of $D$ is given by $X^{\#}=A^{-1} X^{*} A$ where $X^{*}$ is the hermitian transpose of $X$ :

$$
\left[\begin{array}{ll}
x & y \\
u & v
\end{array}\right]^{\#}=\left[\begin{array}{cc}
x^{*} & b u^{*} \\
b^{-1} y^{*} & v^{*}
\end{array}\right]
$$

More general examples (crossed products) will be given later.

\section{Formally Real inVolutions on CROSSED PRODUCTS}

Let $D$ be a central division $F$-algebra with involution $*$ and $K$ a maximal subfield of $D$ such that $K^{*} \subseteq K$. In this section we assume that $K / F$ is a Galois extension and we will write $G$ for its Galois group. In Chapter 4 of [2] it is shown that there exists a normalized cocycle $\Phi: G \times G \rightarrow K \backslash\{0\}$ such that $D$ is isomorphic to the crossed product algebra $(K / F, \Phi)$. By definition, $(K / F, \Phi)$ is a right $K$-vector space with basis $\left(e_{\sigma}\right)_{\sigma \in G}$ and its multiplication is defined by

$$
\mu\left(\sum_{\sigma \in G} e_{\sigma} c_{\sigma}, \sum_{\tau \in G} e_{\tau} d_{\tau}\right)=\sum_{\sigma, \tau \in G} e_{\sigma \tau} \Phi(\sigma, \tau) c_{\sigma}^{\tau} d_{\tau}
$$


The mapping $f: D \rightarrow K$ from Section 2 satisfies

$$
f\left(\sum_{\sigma \in G} e_{\sigma} c_{\sigma}\right)=c_{\mathrm{id}}
$$

Namely, for every $\sigma \neq \mathrm{id}$, there exists $k \in K$ such that $k^{\sigma} \neq k$. Since $k e_{\sigma}=e_{\sigma} k^{\sigma}$ and $f$ is $K-K$ bilinear, it follows that $f\left(e_{\sigma}\right) k=k^{\sigma} f\left(e_{\sigma}\right)$. By the choice of $k, f\left(e_{\sigma}\right)=0$. On the other hand $f\left(e_{\mathrm{id}}\right)=f(1)=1$.

Our first example (motivated by [6]) shows that formal reality of $*$ does not necessarily imply formal reality of its extension to $D \otimes_{F} K$.

Example 2. Let $F=\mathbb{C}(a, b)$ be the field of all complex rational functions in two variables and let $\epsilon=\frac{-1+i \sqrt{3}}{2}$. Let $D_{3}$ be the symbol algebra $A_{\epsilon}(a, b ; F)$, i.e. $D_{3}$ is an $F$-algebra with two generators $x$ and $y$ which satisfy the following relations $x^{3}=a, y^{3}=b, y x=\epsilon x y$. Let $*$ be the involution on $D_{3}$ which fixes $a, b, x, y$ and conjugates the elements from $\mathbb{C}$. Note that $K=F(x)$ is a maximal $*$-invariant subfield of $D_{3}$. We claim that $D_{3}$ is formally real but $D_{3} \otimes K$ is not.

By eliminating $a$ and $b$ using relations $x^{3}=a, y^{3}=b$, we see that $D_{3}$ is the skew field of fractions of the Ore domain $R=\mathbb{C}\langle x, y\rangle /(y x-\epsilon x y)$. Each element from $R$ can be written uniquely as a linear combination of monomials $x^{m} y^{n}$ with complex coefficients. We pick any monomial ordering $<$ and write $\operatorname{lt}(d)$ for the leading term of $d$ with respect to this monomial ordering. If $\operatorname{lt}(d)=c x^{m} y^{n}$, then $\operatorname{lt}\left(d d^{*}\right)=c \bar{c} \epsilon^{2 m n} x^{2 m} y^{2 n}$. Since $\mathbb{C}$ is formally real, it follows that $R$ is formally real as well. Hence, $D_{3}$ is also formally real by Proposition 2 in [1].

The involution \# on $D \otimes_{F} K \cong M_{3}(K)$ which extends $*$ is given by $X^{\#}=A^{-1} X^{*} A$ where $A=\left[f\left(y^{i} y^{j}\right)\right]_{i, j=0,1,2}$; see Proposition $[8$. By the discussion above, $f(1)=1, f(y)=0, f\left(y^{2}\right)=0, f\left(y^{3}\right)=b$ and $f\left(y^{4}\right)=$ 0 . Therefore, $A$ is congruent to the diagonal matrix $\operatorname{diag}(1, b,-b)$. Since there is no unital hermitian cone on $S_{1}(K)$ which contains $1, b$ and $-b,\left(M_{3}(K), \#\right)$ is not formally real by Corollary 4 .

The goal of this section is to characterize involutions on $D$ for which the extended involutions on $D \otimes_{F} K$ are formally real. We need an auxiliary result:

Proposition 9. If $D=(K / F, \Phi)$ is a division algebra with involution * satisfying $K^{*} \subseteq K$, then the following assertions are equivalent:

(1) $\left(k^{*}\right)^{\sigma}=\left(k^{\sigma}\right)^{*}$ for every $k \in K$ and $\sigma \in G$,

(2) $e_{\sigma}^{*} e_{\sigma} \in K$ for every $\sigma \in G$,

(3) $f\left(e_{\tau}^{*} e_{\sigma}\right)=0$ for every $\sigma, \tau \in G$ such that $\sigma \neq \tau$.

Proof. For every $k \in K$ and every $\sigma, \tau \in G$, we have that

$$
\left(k^{\tau}\right)^{*} e_{\tau}^{*} e_{\sigma}=\left(e_{\tau} k^{\tau}\right)^{*} e_{\sigma}=\left(k e_{\tau}\right)^{*} e_{\sigma}=e_{\tau}^{*} k^{*} e_{\sigma}=e_{\tau}^{*} e_{\sigma}\left(k^{*}\right)^{\sigma} .
$$


We will use this identity several times.

$(1) \Rightarrow(2)$ Suppose that $\left(k^{\sigma}\right)^{*}=\left(k^{*}\right)^{\sigma}$ for every $k \in K$ and $\sigma \in G$. Then the identity (used with $\tau=\sigma$ ) implies that $e_{\sigma}^{*} e_{\sigma}$ commutes with $\left(k^{*}\right)^{\sigma}$ for every $k \in K$, hence it commutes with every element from $K$. By the Double Centralizer Theorem, it follows that $e_{\sigma}^{*} e_{\sigma} \in K$ for every $\sigma \in G$.

$(2) \Rightarrow(1)$ If $e_{\sigma}^{*} e_{\sigma} \in K$ for every $\sigma$, then the identity implies that $\left(k^{*}\right)^{\sigma}=\left(k^{\sigma}\right)^{*}$ for every $k \in K$ and $\sigma \in G$.

$(1) \Rightarrow(3)$ Pick any $\sigma, \tau \in G$ such that $\sigma \neq \tau$. Then there exists $k \in K$ such that $\left(k^{\tau}\right)^{*} \neq\left(k^{\sigma}\right)^{*}$. The identity and the assumption imply that $\left(k^{\tau}\right)^{*} f\left(e_{\tau}^{*} e_{\sigma}\right)=f\left(e_{\tau}^{*} e_{\sigma}\right)\left(k^{*}\right)^{\sigma}=f\left(e_{\tau}^{*} e_{\sigma}\right)\left(k^{\sigma}\right)^{*}$, hence $f\left(e_{\tau}^{*} e_{\sigma}\right)=0$.

$(3) \Rightarrow(1)$ Suppose that $f\left(e_{\tau}^{*} e_{\sigma}\right)=0$ for every $\sigma, \tau \in G$ such that $\sigma \neq \tau$. The fact that the matrix $A=\left[f\left(e_{\tau}^{*} e_{\sigma}\right)\right]$ is nonsingular implies that $f\left(e_{\sigma}^{*} e_{\sigma}\right) \neq 0$ for every $\sigma \in G$. Replacing $\tau$ by $\sigma$ in the identity and applying $f$, we get that $\left(k^{\sigma}\right)^{*} f\left(e_{\sigma}^{*} e_{\sigma}\right)=f\left(e_{\sigma}^{*} e_{\sigma}\right)\left(k^{*}\right)^{\sigma}$. Since $f\left(e_{\sigma}^{*} e_{\sigma}\right) \neq$ 0 , it follows that $\left(k^{\sigma}\right)^{*}=\left(k^{*}\right)^{\sigma}$ for every $k \in K$ and $\sigma \in G$.

Theorem 10. If $D=(K / F, \Phi)$ is a division algebra with involution * satisfying $K^{*} \subseteq K$, then the following assertions are equivalent:

(1) $D \otimes K$ is formally real,

(2) $D$ is formally real and $\left(k^{*}\right)^{\sigma}=\left(k^{\sigma}\right)^{*}$ for every $k \in K$ and $\sigma \in G$,

(3) $e_{\sigma}^{*} e_{\sigma} \in K$ for every $\sigma \in G$ and there exists a unital hermitian cone on $K$ which contains all of them.

Proof. (1) $\Rightarrow(2)$ Suppose that $D \otimes K$ is formally real. We claim that $f\left(e_{\sigma}^{*} e_{\sigma}\right) \neq 0$ for every $\sigma$. The identity from the proof of Proposition 9 (used with $\tau=\sigma$ ) then implies that $\left(k^{\sigma}\right)^{*}=\left(k^{*}\right)^{\sigma}$ for every $k \in K$ and $\sigma \in G$. If the claim is false then the matrix $A$ has a zero entry on the diagonal, hence it is congruent to a diagonal matrix which has two nonzero entries of opposite signs. Hence $A$ cannot belong to a unital hermitian cone on $\left(M_{n}(K), *\right)$. Therefore $(D, *) \cong\left(M_{n}(K)\right.$, \#) does not have a unital hermitian cone, contrary to the assumption. The second part of assertion (2) follows from the fact that $D$ is contained in $D \otimes K$.

$(2) \Rightarrow(3)$ If $(2)$ is true, then by Proposition $9 e_{\sigma}^{*} e_{\sigma} \in K$ for every $\sigma \in G$. Since $D$ is formally real, the set of all sums of hermitian squares is a unital hermitian cone which contains $e_{\sigma}^{*} e_{\sigma}$ and it restricts to a unital hermitian cone on $K$.

$(3) \Rightarrow(1)$ If (3) is true, then by the proposition $A=\left[f\left(e_{\tau}^{*} e_{\sigma}\right)\right]$ is a diagonal matrix with entries $a_{\sigma}=e_{\sigma}^{*} e_{\sigma}$. By the assumption there exists a unital hermitian cone on $K$ containing all $a_{\sigma}$. Hence $D \otimes K$ is formally real by Corollary 4 , 
For the sake of completeness we also note that $(D, *)$ need not be formally real even if all its maximal $*$-subfields are formally real.

Example 3. We consider a variant of Example 2. Let $\epsilon=\frac{-1+i \sqrt{3}}{2}$ and $a=b=2$. Let $D$ be a $\mathbb{Q}(\epsilon)$-algebra generated with two generators $x, y$ and three relations $x^{3}=a, y^{3}=b, y x=\epsilon x y$. The involution is defined by $\epsilon^{*}=\epsilon^{-1}, x^{*}=x, y^{*}=y$.

We claim that every maximal $*$-subfield of $D$ is formally real. Note that $\left.*\right|_{\mathbb{Q}(\epsilon)} \neq \mathrm{id}$, hence we know by the proof of Lemma 6 that every maximal *-subfield of $D$ can be generated by a symmetric element. It follows that every maximal $*$-subfield can be $*$-embedded into $\mathbb{C}$ with standard involution, thus it is formally real.

We also claim that $D$ is not formally real. It suffices to see that

$$
d_{1}^{*} d_{1}+d_{2}^{*} d_{2}+d_{3}^{*} d_{3}+d_{4}^{*} d_{4}=0,
$$

where

$$
\begin{array}{ll}
d_{1}=\epsilon^{-1} x+x^{2}+2 y, & d_{3}=2 x-x^{2}+x y^{2}, \\
d_{2}=1-\epsilon^{-1} x y-x^{2} y^{2}, & d_{4}=3-x-x^{2} .
\end{array}
$$

\section{Extensions And CONTRACTIONS OF UNITAL HERMITIAN CONES}

Let $D=(K / F, \Phi)$ be a crossed product division algebra with involution $*$ such that $K^{*} \subseteq K$ and $(D \otimes K, *) \cong\left(M_{n}(K), \#\right)$ is formally real. Let $\left(e_{\phi}\right)_{\phi \in G}$ be the standard right $K$-basis of $D$ and let $\lambda: D \rightarrow M_{n}(K)$ be the left regular representation of $D$ with respect to the standard basis. Let $\mathcal{N}$ be the set of all unital hermitian cones on $(K, *)$ which contain $a_{\sigma}=e_{\sigma}^{*} e_{\sigma}$ for every $\sigma \in G$. By assertion (3) of Theorem 10, $\mathcal{N}$ is nonempty.

Lemma 11. We can define an action of $G$ on $\mathcal{N}$ by

$$
N_{\sigma}=\left(\frac{1}{a_{\sigma}} N\right)^{\sigma^{-1}}=\left\{\left(\frac{n}{a_{\sigma}}\right)^{\sigma^{-1}} \mid n \in N\right\} .
$$

Proof. An element $k \in K$ belongs to $N_{\sigma}$ if and only if $a_{\sigma} k^{\sigma} \in N$. It follows that $N_{\sigma}+N_{\sigma} \subseteq N_{\sigma}$ and $N_{\sigma} \cap-N_{\sigma}=\{0\}$. By assertion (2) of Theorem 10, $\left.*\right|_{K}$ commutes with every element of $G$. It follows that $N_{\sigma} \subseteq S_{1}(K)$ and $r^{*} N_{\sigma} r \subseteq N_{\sigma}$ for every $r \in K$. For every $\sigma, \tau \in G$ we have $a_{\sigma} a_{\tau}^{\sigma}=e_{\sigma}^{*} e_{\tau}^{*} e_{\tau} e_{\sigma}=\Phi(\tau, \sigma)^{*} a_{\tau \sigma} \Phi(\tau, \sigma) \in N$. It follows that $N_{\sigma}$ contains $a_{\tau}$ for every $\tau \in G$ and that $\left(N_{\tau}\right)_{\sigma}=N_{\tau \sigma}$.

Let $\mathcal{M}$ be the set of all unital hermitian cones on $(D, *)$. For every $M \in \mathcal{M}$ write $M^{c}=M \cap K$ and note that $M^{c} \in \mathcal{N}$. For every $N \in \mathcal{N}$ write $N^{e}=\lambda^{-1} \phi^{-1} F(N)=\left\{c \in D \mid x^{*} A \lambda(c) x \in N\right.$ for every $\left.x \in K^{n}\right\}$ where $A=\operatorname{diag}\left(a_{\sigma}\right)_{\sigma \in G}$. By Corollary 4, we see that $\phi^{-1} F(N)$ is a unital hermitian cone on $\left(M_{n}(K)\right.$, \#), hence $N^{e} \in \mathcal{M}$. 
Theorem 12. Setup from above. For every $N \in \mathcal{N}$ we have

(1) $N^{e}=\left\{u \in D \mid f\left(d^{*} u d\right) \in N\right.$ for every $\left.d \in D\right\}$,

(2) $N^{e c}=\bigcap_{\sigma \in G} N_{\sigma}$,

(3) $\left(N_{\sigma}\right)^{e}=N^{e}$ for every $\sigma \in G$,

(4) $N^{e c e}=N^{e}$.

For every $M \in \mathcal{M}$ we have

(5) $\left(M^{c}\right)_{\sigma}=M^{c}$ for every $\sigma \in G$,

(6) $M^{c e c}=M^{c}$.

Proof. To prove (1), pick any $u \in D$ and note that $\left[e_{\sigma}^{*} u e_{\tau}\right]_{\sigma, \tau \in G}=$ $\left[e_{\sigma}^{*} e_{\tau}\right]_{\sigma, \tau \in G} \lambda(u)$. Applying $f$ to all $n^{2}$ equations, we get $\left[f\left(e_{\sigma}^{*} u e_{\tau}\right)\right]_{\sigma, \tau \in G}=$ $\left[f\left(e_{\sigma}^{*} e_{\tau}\right)\right]_{\sigma, \tau \in G} \lambda(u)$ which is equal to $A \lambda(u)$. For every $x=\left(k_{\phi}\right)_{\phi \in G}$, we get $x^{*} A \lambda(u) x=f\left(d^{*} u d\right)$, where $d=\sum_{\phi \in G} e_{\phi} k_{\phi}$. Claim (1) now follows from the definition of $N^{e}$.

Claim (3) is rather tricky. Pick $\sigma \in G$ and $u=\sum_{\omega \in G} e_{\omega} r_{\omega} \in D$. For every $d=\sum_{\phi \in G} e_{\phi} k_{\phi} \in D$ write $d_{\sigma}=\sum_{\phi \in G} e_{\phi \sigma} \Phi(\phi, \sigma) k_{\phi}^{\sigma}$. We have

$$
\begin{aligned}
f\left(d_{\sigma}^{*} u d_{\sigma}\right) & =\sum_{\omega} f\left(d_{\sigma}^{*} e_{\omega} r_{\omega} d_{\sigma}\right) \\
& =\sum_{\omega, \phi, \tau} f\left(k_{\phi}^{\sigma *} \Phi(\phi, \sigma)^{*} e_{\phi \sigma}^{*} e_{\omega} r_{\omega} e_{\tau \sigma} \Phi(\tau, \sigma) k_{\tau}^{\sigma}\right) \\
& =\sum_{\omega, \phi, \tau} f\left(k_{\phi}^{\sigma *} \Phi(\phi, \sigma)^{*} e_{\phi \sigma}^{*} e_{\omega \tau \sigma} r_{\omega}^{\tau \sigma} \Phi(\omega, \tau \sigma) \Phi(\tau, \sigma) k_{\tau}^{\sigma}\right) \\
& \stackrel{(*)}{=} \sum_{\omega, \phi, \tau} k_{\phi}^{\sigma *} \Phi(\phi, \sigma)^{*} a_{\phi \sigma} \delta_{\phi, \omega \tau} r_{\omega}^{\tau \sigma} \Phi(\omega \tau, \sigma) \Phi(\omega, \tau)^{\sigma} k_{\tau}^{\sigma} \\
& =\sum_{\omega, \tau} k_{\omega \tau}^{\sigma *} \Phi(\omega \tau, \sigma)^{*} a_{\omega \tau \sigma} r_{\omega}^{\tau \sigma} \Phi(\omega \tau, \sigma) \Phi(\omega, \tau)^{\sigma} k_{\tau}^{\sigma} \\
& \stackrel{(*)}{=} \sum_{\omega, \tau} k_{\omega \tau}^{* \sigma} a_{\sigma} a_{\omega \tau}^{\sigma} r_{\omega}^{\tau \sigma} \Phi(\omega, \tau)^{\sigma} k_{\tau}^{\sigma} \\
& =a_{\sigma}\left(\sum_{\omega, \tau} k_{\omega \tau}^{*} a_{\omega \tau} r_{\omega}^{\tau} \Phi(\omega, \tau) k_{\tau}\right)^{\sigma} .
\end{aligned}
$$

At (*) we used the cocycle identity and $f\left(e_{\phi \sigma}^{*} e_{\omega \tau \sigma}\right)=a_{\phi \sigma} \delta_{\phi, \omega \tau}$. At (**) we used $k_{\omega \tau}^{\sigma *}=k_{\omega \tau}^{* \sigma}$ and $\Phi(\omega \tau, \sigma)^{*} a_{\omega \tau \sigma} \Phi(\omega \tau, \sigma)=a_{\sigma} a_{\omega \tau}^{\sigma}$. For $\sigma=\mathrm{id}$, we get $f\left(d^{*} u d\right)=\sum_{\omega, \tau} k_{\omega \tau}^{*} a_{\omega \tau} r_{\omega}^{\tau} \Phi(\omega, \tau) k_{\tau}$, hence

$$
f\left(d_{\sigma}^{*} u d_{\sigma}\right)=a_{\sigma} f\left(d^{*} u d\right)^{\sigma} .
$$

If $u \in N^{e}$, then $\lambda\left(d^{*} u d\right) \in N$ for every $d \in D$. It follows that $a_{\sigma} f\left(d^{*} u d\right)^{\sigma}=f\left(d_{\sigma}^{*} u d_{\sigma}\right) \in N$ for every $d \in D$. Hence, $f\left(d^{*} u d\right) \in N_{\sigma}$ for every $d \in D$, which implies that $u \in N_{\sigma}^{e}$. Since every element of $D$ is of the form $d_{\sigma}$, we can also prove the opposite inclusion.

To prove (2), pick $k \in K$ and note that $k \in N^{e}$ if and only if $\operatorname{diag}\left(a_{\sigma} k^{\sigma}\right)_{\sigma \in G}=A \lambda(k)$ belongs to $F(N)$. Hence, $k \in N^{e}$ if and only if $a_{\sigma} k^{\sigma} \in N$ for every $\sigma \in G$. Claim (2) now follows from the definition of $N_{\sigma}$. Claim (4) is a simple consequence of claims (2) and (3).

Claim (5) follows from the fact that an element $k \in K$ belongs to $M^{c}$ if and only if $a_{\sigma} k^{\sigma}=e_{\sigma}^{*} k e_{\sigma} \in M^{c}$. Claim (6) now follows from Claim (2) applied to $N=M^{c}$. 
Let us state two simple corollaries.

Corollary 13. Setup from Theorem 12. For every unital hermitian cone $N$ on $K$, the following are equivalent:

(1) $a_{\sigma} n^{\sigma} \in N$ for every $n \in N$ and $\sigma \in G$ (i.e. $N \in \mathcal{N}$ and $N_{\sigma}=N$ for every $\sigma \in \sigma$ ),

(2) $N$ extends to a unital hermitian cone on $D$ (i.e. $N=M \cap K$ for some unital hermitian cone $M$ on $D)$,

(3) $N$ extends to a unital hermitian cone on $\left(M_{n}(K)\right.$, \#) (i.e. $N=$ $\lambda^{-1}(L) \cap K$ for some unital hermitian cone $L$ on $\left(M_{n}(K), \#\right)$.)

Proof. Clearly (3) implies (2). By Claim (5) of Theorem 12, (2) implies (1). To see that (1) implies (3) take $L=\phi^{-1} F(N)$. By Corollary 4 , we see that $L$ is a unital hermitian cone on $\left(M_{n}(K)\right.$, \#). Note that $\lambda^{-1}(L)=N^{e}$ by the definition of $N^{e}$, hence $\lambda^{-1}(L) \cap K=N^{e c}$. By assumption $N=N_{\sigma}$ for every $\sigma \in G$. Hence $N^{e c}=\bigcap_{\sigma \in G} N_{\sigma}=N$ by Claim (2) of Theorem 12 .

Corollary 14. Setup from Theorem 12. For every unital hermitian cone $M$ on $D$ the following are equivalent:

(1) $M$ extends to a unital hermitian cone on $\left(M_{n}(K)\right.$,\#) (i.e. $M=$ $\lambda^{-1}(L)$ for some unital hermitian cone $L$ on $\left.\left(M_{n}(K), \#\right)\right)$,

(2) An element $u$ of $D$ belongs to $M$ if and only if $f\left(d^{*} u d\right) \in M$ for every $d \in D$ (i.e. $M=M^{c e}$ ).

Proof. Suppose that (1) is true. By Theorem 3 and Corollary 4 , there exists a unital hermitian cone $N$ on $K$ containing all $a_{\phi}, \phi \in G$ such that $L=\phi^{-1} F(N)$. It follows that $M=N^{e}$ for some $N \in \mathcal{N}$. Hence $M^{c e}=N^{e c e}=N^{e}=M$ by Claim (5) of Theorem 12, Now (2) follows from Claim (1) of Theorem 12 (with $N=M^{c}$ ).

If (2) is true, then $M=M^{c e}$ by Claim (1) of Theorem 12 (with $\left.N=M^{c}\right)$. You can take $L=\phi^{-1} F\left(M^{c}\right)$ to get (1).

The following question remains open: Is $M^{c e} \subseteq M$ for every $M \in$ $\mathcal{M}$ ?

We finish this section with two examples which prove the following claims:

(1) a unital hermitian cone from $\mathcal{N}$ can have no extension to $D$,

(2) a unitial hermitian cone from $\mathcal{N}$ can have two different extensions to $D$,

(3) a unital hermitian cone on $D$ can have no extension to $D \otimes_{F} K$,

(4) a unital hermitian cone on $D$ can have two different extension to $D \otimes_{F} K$. 
Example 4. Let $D=\left(\frac{a, b}{\mathbb{Q}}\right)$ where $a, b>0$ and $i^{*}=i, j^{*}=j$. Then $K=\mathbb{Q}(i)$ can be identified with $\mathbb{Q}(\sqrt{a}) \subset \mathbb{R}$, where either $\sqrt{a}>0$ or $\sqrt{a}<0$. The ordering of $\mathbb{R}$ then induces unital hermitian cones (in fact orderings) $N_{1}$ and $N_{2}$ on $K$, such that $i \in N_{1}$ and $-i \in N_{2}$. Since $b>0, b$ is a sum of four squares, hence both $N_{1}$ and $N_{2}$ contain $a_{2}=b$. However, neither $N_{1}$ nor $N_{2}$ extends to $D$. Suppose that $N_{1}$ extends to a unital hermitian cone $M_{1}$ of $D$. Since $i \in N_{1}$, it follows that $j^{*} i j \in M_{1}$, so that $-b i=j^{*} i j \in M_{1} \cap K=N_{1}$. Since $1 / b$ is a sum of squares, it follows that $-i \in N_{1}$, a contradiction. The proof that $N_{2}$ does not extend to $D$ is analogous. This proves Claim (1) above. Note that by Corollary $13 N_{1} \cap N_{2}$ extends to $D$.

Theorem 3 and Corollary 4 tell us that $L_{1}=\phi^{-1}\left(F\left(N_{1}\right)\right)$ and $L_{2}=$ $\phi^{-1}\left(F\left(N_{2}\right)\right)$ are unital hermitian cones on $\left(M_{n}(K), \#\right)$. Since $N_{1} \neq N_{2}$, also $L_{1} \neq L_{2}$. Assertion (3) of Theorem 12 implies that $\lambda^{-1}\left(L_{1}\right)=$ $\lambda^{-1}\left(L_{2}\right)$. This proves Claim (4) above.

Example 5. Write $F=\mathbb{R}(a, b), D=\left(\frac{a, b}{F}\right)$ and $K=F(i)$. The involution on $D$ is defined by $i^{*}=i$ and $j^{*}=j$. Let $R$ be the $\mathbb{R}$ subalgebra of $D$ generated by $i$ and $j$. Note that $R=\mathbb{R}\langle i, j\rangle /(i j+j i)$ is an Ore domain and that its skew field of fractions is $D$. Similarly, the fields of fractions of commutative $\mathbb{R}$-subalgebras $S=\mathbb{R}\left[i, j^{2}\right]$ and $T=\mathbb{R}\left[i^{2}, j^{2}\right]$ are $K$ and $F$ respectively. We will construct two different unital hermitian cones $M_{1}$ and $M_{2}$ on $R$ such that $M_{1} \cap S=M_{2} \cap S$. By Proposition 2 in [1], $M_{1}$ and $M_{2}$ extend uniquely from $R$ to $D$. These extensions are clearly different, but they have the same restriction to $K$. This will prove Claim (2) above.

Every element of $R$ is a linear combination of monomials $i^{m} j^{n}$. We pick any monomial ordering $<$ and write $\operatorname{lt}(d)$ for the leading term of $d$ with respect to this monomial ordering. If $s \in R$ is symmetric and $\operatorname{lt}(s)=c i^{m} j^{n}$, then $2 \mid m n$. For every $r$ such that $\operatorname{lt}(r)=u i^{k} j^{l}$ we have that $\operatorname{lt}\left(r^{*}(-1)^{\frac{m n}{2}} s r\right)=c u^{2}(-1)^{\frac{(m+2 k)(n+2 l)}{2}} i^{m+2 k} j^{n+2 l}$. It follows that

$$
\begin{gathered}
M_{1}=\left\{s \in \operatorname{Sym}(R) \mid \operatorname{lt}(s)=c i^{m} j^{n} \text { where } c(-1)^{\frac{m n}{2}} \geq 0\right\} \text { and } \\
M_{2}=\left\{s \in \operatorname{Sym}(R) \mid \operatorname{lt}(s)=c i^{m} j^{n} \text { where } c(-1)^{\frac{m n}{2}+n} \geq 0\right\}
\end{gathered}
$$

are unital hermitian cones (even Baer orderings) on $R$. Clearly, they are different and they have the same restriction to $S$ (take $n$ even).

Note that $j \in M_{1}$ and $-j \in M_{2}$. We will show that there is no unital hermitian cone on $D \otimes_{F} K$ which contains either $\lambda(j)$ or $-\lambda(j)$. Therefore, neither $M_{1}$ nor $M_{2}$ extends to $D \otimes_{F} K$. This will prove 
Claim (3) above. Note that

$$
A \lambda(j)=\left[\begin{array}{ll}
1 & 0 \\
0 & b
\end{array}\right]\left[\begin{array}{ll}
0 & b \\
1 & 0
\end{array}\right]=\left[\begin{array}{ll}
0 & b \\
b & 0
\end{array}\right] \text { is congruent to }\left[\begin{array}{cc}
-b & 0 \\
0 & b
\end{array}\right] .
$$

Clearly, there is no unital hermitian cone on $K$ which contains $b$ and $-b$. Therefore, by Corollary 4 , there is no unital hermitian cone on $\left(M_{n}(K), \#\right)$ which contains $\lambda(j)$. The same proof also works for $-\lambda(j)$.

\section{HeRmitian TRACE FORMS}

Let $A$ be a central simple $F$-algebra with involution $*$ and $\operatorname{tr}: A \rightarrow F$ its reduced trace. The mapping

$$
a \mapsto \operatorname{tr}\left(a^{*} a\right)
$$

is called the hermitian trace form of $(A, *)$. Write $N_{(A, *)}$ for the image of this map. We say that the hermitian trace form is positive semidefinite if $N_{(A, *)} \cap-N_{(A, *)}=\{0\}$. In this case, $N_{(A, *)}$ is a unital hermitian cone on $F$.

Proposition 15. Let $D$ be a central division algebra over $F$ with involution $*$ and let $K$ be a maximal $*$-invariant subfield. Consider the following assertions:

(1) There exists a *-ordering (=multiplicatively closed unital hermitian cone) on $F$ which contains $N_{(D, *)}$.

(2) The extension of the hermitian trace form to $\left(D \otimes_{F} K, *\right)$ is positive semidefinite.

(3) The hermitian trace form on $(D, *)$ is positive semidefinite.

(4) $(D \otimes K, *)$ is formally real, i.e. has a unital hermitian cone.

(5) $(D, *)$ is formally real.

Then $(1) \Rightarrow(2) \Rightarrow(3) \Rightarrow(4) \Rightarrow(5)$.

Proof. Implications $(2) \Rightarrow(3)$ and $(4) \Rightarrow(5)$ are clear.

$(1) \Rightarrow(2)$ Let $P$ be the $*$-ordering containing $N=N_{(D, *)}$. Write $M_{P}=\left\{c \in \operatorname{Sym}(K) \mid \forall k \in K: \operatorname{tr}\left(k^{*} c k\right) \in P\right\}$. To see that $N_{(D \otimes K, *)} \cap$ $-N_{(D \otimes K, *)}=\{0\}$, it suffices to show that $M_{P} \cap-M_{P}=\{0\}$ and $N_{(D \otimes K, *)} \subseteq M_{P}$. If $c \in M_{P} \cap-M_{P}$, then $\operatorname{tr}\left(k^{*} c k\right)=0$ for every $k \in K$. If $c \neq 0$, then write $c^{-1}=k_{1}^{*} k_{1}-k_{2}^{*} k_{2}$ for $k_{1}=\frac{1+c^{-1}}{2}$ and $k_{2}=\frac{1-c^{-1}}{2}$ and note that $\operatorname{tr}(1)=\operatorname{tr}\left(k_{1}^{*} c k_{1}\right)-\operatorname{tr}\left(k_{2}^{*} c k_{2}\right)=0$ which is impossible because char $F \neq 0$ by the existence of $P$. It follows that $M_{P}$ is a unital hermitian cone on $(K, *)$. Next we show that $N \subseteq M_{P}$. To see this, pick $c \in N$ and note that $\operatorname{tr}\left(k^{*} c k\right)=c \operatorname{tr}\left(k^{*} k\right) \in N \cdot N \subseteq P$ for every $k \in K$. Now we can prove that $N_{(D \otimes K, *)} \subseteq M_{P}$. Pick an $F$-basis $g_{1}, \ldots, g_{n}$ of $D$ such that $\left[\operatorname{tr}\left(g_{i}^{*} g_{j}\right)\right]_{i, j}$ is diagonal. By the definition 
of $N$, the diagonal elements $\operatorname{tr}\left(g_{i}^{*} g_{i}\right)$ belong to $N$. For every element $z \in D \otimes K \cong M_{n}(K)$ there exist $k_{i} \in K$ such that $z=\sum_{i=1}^{n} \lambda\left(g_{i}\right) k_{i}$. It follows that $\operatorname{tr}\left(z^{\#} z\right)=\sum_{i=1}^{n} k_{i}^{*} k_{i} \operatorname{tr}\left(g_{i}^{*} g_{i}\right) \in M_{P}$, because $M_{P}$ is a unital hermitian cone on $K$ which contains $N$.

$(3) \Rightarrow(4)$ Let $N=N_{(D, *)}$ and $M_{N}=\left\{c \in K \mid \forall k \in K: \operatorname{tr}\left(k^{*} c k\right) \in\right.$ $N\}$. As above, we see that $M_{N} \cap-M_{N}=\{0\}$ hence $M_{N}$ is a unital hermitian cone on $(K, *)$. Let $e_{i}, i=1, \ldots, m$ be a right $K$-basis

of $D$ such that $\left[f\left(e_{i}^{*} e_{j}\right)\right]_{i, j}$ is diagonal and write $a_{i}=f\left(e_{i}^{*} e_{i}\right)$. Since $\operatorname{tr}\left(k^{*} a_{i} k\right)=\operatorname{tr}\left(f\left(\left(e_{i} k\right)^{*}\left(e_{i} k\right)\right)\right)=\operatorname{tr}\left(\left(e_{i} k\right)^{*}\left(e_{i} k\right)\right) \in N$ for every $k \in K$, it follows that $a_{i} \in M_{N}$ for every $i=1, \ldots, m$. Now Corollary 4 implies that $D \otimes K$ is formally real.

In Section 5 of [7], it is proved that the five assertions of the proposition are equivalent for quaternion algebras $\left(\frac{a, b}{F}\right)$ with standard involution. In Section 4 of [5], it is shown that (5) is equivalent to (3) in many other cases. (They use different terminology.) By our Example 2. (5) is not always equivalent to (4). We conjecture that in general any two assertions are inequivalent.

Acknowledgements: I would like to thank to Prof. Tom Craven for sharing with me his experience. He proved an early version of Theorem 11 (with $\epsilon=1$ and $*=\mathrm{id}$ ) and read later versions of the manuscript. I would also like to thank to Dr. Thomas Unger for his comments on Section 5 .

\section{REFERENCES}

[1] Cimprič, J. Maximal quadratic modules on *-rings. To appear in Algebr. Represent. Theory.

[2] Herstein, I. N. "Noncommutative rings." The Carus Mathematical Monographs, No. 15, Math. Assoc. Amer., New York 1968, xi+199 pp.

[3] Knus, M.-A. "Quadratic and Hermitian forms over rings." With a foreword by I. Bertuccioni. Grundlehren der Mathematischen Wissenschaften [Fundamental Principles of Mathematical Sciences], 294. Springer-Verlag, Berlin, 1991. xii+524 pp.

[4] Knus, M.-A.; Merkurjev, A.; Rost, M.; Tignol, J.-P. "The book of involutions. With a preface in French by J. Tits." American Mathematical Society Colloquium Publications, 44. American Mathematical Society, Providence, RI, 1998. xxii+593 pp.

[5] Lewis, D. W.; Scheiderer, C.; Unger, T. A weak Hasse principle for central simple algebras with an involution. Proceedings of the Conference on Quadratic Forms and Related Topics (Baton Rouge, LA, 2001). Doc. Math. 2001, Extra Vol., 241-251 (electronic).

[6] Morandi, P. J.; Sethuraman, B. A. Noncrossed product division algebras with a Baer ordering. Proc. Amer. Math. Soc. 123 (1995), no. 7, 1995-2003. 
[7] Procesi, C.; Schacher, M. A non-commutative real Nullstellensatz and Hilbert's 17th problem. Ann. of Math. (2) 104 (1976), no. 3, 395-406.

Jaka Cimprič, University of Luubljana, Faculty of Math. and Phys., Dept. of Math., Jadranska 19, SI-1000 Luubluana, SloveniJa. E-mail: CIMPRIC@FMF.UNI-LJ.SI. WWW PAGE: HTTP://WWW.FMF.UNI-LJ.SI//CIMPRIC. 